\title{
ASOCIACIÓN ENTRE LOS POLIMORFISMOS DE LOS GENES DE LA ENZIMA CONVERTIDORA DE ANGIOTENSINA Y LOS RECEPTORES AT1R Y AT2R Y EL CÁNCER DE MAMA. ESTUDIO DE CASOS Y CONTROLES
}

\author{
The association between the angiotensin converter \\ enzyme (ACE), AT1R and AT2R receptor gene \\ polymorphisms and breast cancer: a case control \\ study
}

\author{
Andrés Felipe Castro D*, Nelsy Loango, M.Sc.**, Bayron Ruíz, M.D., M.Sc.***, \\ Patricia Landázuri, M.Sc., Ph.D.****
}

Recibido: junio 18/10 - Aceptado: enero 31/11

\section{RESUMEN}

Introducción: el cáncer de mama es el segundo tipo de cáncer más común y la quinta causa de muerte por cáncer a nivel mundial. Un candidato potencial para la terapia de quimioprevención es el sistema renina-angiotensina en sus componentes: la enzima convertidora de la angiotensina (ECA), y los receptores de angiotensina II tipo 1 y 2 (AT1R y AT2R).

Objetivo: establecer la asociación entre los polimorfismos de los genes de la ECA, los receptores AT1R y AT2R y el cáncer de mama.

* Biólogo, Joven Investigador de COLCIENCIAS. Grupo de Investigación en Enfermedades Cardiovasculares y Metabólicas, Universidad del Quindío. Armenia (Colombia).

* Grupo de Investigación en Enfermedades Cardiovasculares y Metabólicas. Licenciada en Biología, docente Programa de Biología, Facultad de Ciencias Básicas y Tecnologías, Universidad del Quindío. Armenia (Colombia).

** Gineco-obstetra. Grupo de Estudio en Parasitología Molecular. Docente, Programa de Medicina, Facultad Ciencias de la Salud, Universidad del Quindío. Armenia (Colombia).

*** Grupo de Investigación en Enfermedades Cardiovasculares y Metabólicas. Docente, Programa de Medicina, Facultad Ciencias de la Salud, Universidad del Quindío. Armenia (Colombia). Correo electrónico: plandazu@uniquindio.edu.co
Materiales y métodos: se incluyeron 50 casos y 50 controles y se genotipificaron los polimorfismos genéticos.

Resultados: el genotipo II presentó mayor frecuencia en los controles que en los casos, mientras que el genotipo ID fue más frecuente en los casos, sin diferencias estadísticamente significativas. No se encontró ningún tipo de efecto aditivo de los genotipos y el riesgo de cáncer de mama en nuestra población de estudio.

Conclusiones: se ha descrito que la susceptibilidad al cáncer de mama es un fenómeno complejo, en el cual múltiples genes pueden desempeñar un papel importante. Para el caso de esta población, los polimorfismos evaluados no se asocian al cáncer de mama. Se requieren más estudios, con mayor fuerza metodológica sobre este tema, antes de proponer cambios en la práctica clínica.

Palabras clave: cáncer, enzima convertidora de angiotensina (ECA), receptores de angiotensina, polimorfismo genético. 


\section{SUMMARY}

Introduction: breast cancer is the second most common type of cancer and the fifth cause of death due to cancer around the world. The components of the renin-angiotensin system are potential candidates for chemoprevention therapy: the angiotensin converter enzyme (ACE) and type 1 and 2 angiotensin II receptors (AT1R and AT2R).

Objective: establishing an association between ACE gene polymorphisms, AT1R and AT2R receptors and breast cancer.

Materials and methods: 50 cases and 50 controls were included and their genetic polymorphisms genotyped.

Results: genotype II occurred with greater frequency in controls than in the cases whilst the ID genotype had greater frequency in the cases (without having statistically significant differences). No type of additive effect of genotypes was found with the risk of breast cancer in the population being studied.

Conclusions: susceptibility to breast cancer has been described as being a complex phenomenon in which many genes may play an important role. In the case of the population studied here, the polymorphisms evaluated were not associated with breast cancer. Further studies having greater methodological rigor regarding this topic are required before changes in clinical practice can be proposed.

Key words: cancer, angiotensin converter enzyme (ACE), angiotensin receptor, genetic polymorphism.

\section{INTRODUCCIÓN}

El cáncer de mama es el segundo tipo de cáncer más frecuente después del cáncer de pulmón y la quinta causa de muerte por cáncer a nivel mundial, con 1 millón de nuevos casos diagnosticados por año ${ }^{1}$ y una incidencia global anual ajustada por edad de 127,8 por cada 100.000. ${ }^{2}$ En Colombia, en el año 2006, se presentaron 1.909 muertes por cáncer de mama, para una tasa cruda de $8,7 \times 100.000 ;^{3}$ esta entidad se considera de etiología multifactorial. ${ }^{4}$ Entre los factores que confieren susceptibilidad genética, se destaca la presencia de mutaciones en los genes supresores tumorales BRCA1, BRCA2 ${ }^{5} \mathrm{y}$ más recientemente en los genes del Sistema Renina Angiotensina (SRA), ante el papel preponderante que desempeña la angiotensina II (AII) en la neovascularización, la proliferación celular, la inflamación y la adhesión celular, lo que le otorga relevancia en el proceso de la carcinogénesis. ${ }^{6}$ Dado que la AII es sintetizada por la acción de la enzima convertidora de angiotensina (ECA) sobre la angiotensina I, en la última década se ha incrementado el estudio del papel que desempeña el gen de la ECA, los inhibidores de la enzima y los bloqueadores de los receptores en la génesis y progresión del cáncer. ${ }^{7}$ Sin embargo, los resultados a la fecha son contradictorios. Otra alternativa más reciente es el estudio de las variaciones genéticas de la ECA y de los receptores de la AII tipo 1 (AT1R) y tipo 2 (AT2R). En el gen de la ECA, se presenta el polimorfismo de Inserción/Deleción (I/D) consistente en la presencia o ausencia de 287 pares de bases en el intrón $16 .{ }^{8}$ Puesto que los niveles plasmáticos de la AII se correlacionan con niveles de ECA, se ha generado la hipótesis según la cual el polimorfismo I/D puede ser importante en la susceptibilidad al cáncer; específicamente el genotipo DD ha sido asociado con riesgo de cáncer de mama. ${ }^{6}$

En el gen AT1R, se ha identificado variación genética que corresponde a una sustitución de adenina por una citosina en la posición 1166 (A1166C) hacia el extremo 3,, 89 que ha sido sujeto de estudio en cuanto a su relación de riesgo mayor de padecer cáncer. ${ }^{10}$ Por otra parte, el receptor AT2R actúa como antagonista de las acciones de AII sobre el AT1R, razón por la que una variación en el gen AT2R que comprende el polimorfismo intrónico +1675G/A (sustitución de un aminoácido Gly-21Val), ${ }^{9}$ ha sido incluido en varios estudios para valorar su influencia en la enfermedad cardiovascular, pero no en cáncer. ${ }^{11}$ 
El objetivo del presente estudio fue establecer la asociación entre el polimorfismo I/D de la ECA, el A1166C en AT1R y el +1675G/A en AT2R con el cáncer de mama como factores de riesgo para el desarrollo de esta entidad.

\section{MATERIALES Y MÉTODOS}

Estudio de casos y controles, en 100 mujeres residentes en el departamento del Quindío, ubicado en la región central de Colombia. Se definió como casos a las mujeres con diagnóstico de cáncer de mama, confirmado histológicamente, que consultaron a la IPS Oncólogos de Occidente, centro de atención especializado para atención de pacientes con cáncer afiliados al aseguramiento privado y la medicina prepagada. Se incluyeron todas las mujeres que consultaron en el período agosto-diciembre de 2009 y que no estaban en proceso de diagnóstico o tratamiento por otro cáncer a la fecha de la recolección de la información. Los controles fueron mujeres con mamografías negativas (BIRADS 1 y 2) tomadas en los 30 días previos en el Centro Radiológico del Quindío (centro de diagnóstico imagenológico). Se realizó muestreo secuencial por conveniencia. El estudio fue aprobado por el Comité de Bioética de la Facultad Ciencias de la Salud de la Universidad del Quindío y del Centro de Oncólogos de Occidente S.A. Todas las participantes diligenciaron de manera voluntaria un consentimiento informado y una encuesta demográfica básica.

\section{Genotipificación}

A cada mujer se le tomó una muestra de sangre por veno-punción ( $5 \mathrm{ml}$ ) en tubo con anticoagulante por personal capacitado para ello, la cual fue transportada al Laboratorio de Bioquímica y Genética de la Universidad del Quindío para su procesamiento. La extracción de ADN se hizo utilizando el estuche comercial Wizard ${ }^{\circledR}$ Genomics (Promega) siguiendo las indicaciones del fabricante.

Para determinar los polimorfismos, se utilizó la reacción en cadena de la polimerasa (PCR). El polimorfismo I/D del gen de la ECA se realizó utilizando 10 pmoles de cada primer: F 5'-CTG GAG ACC ACT CCC ATC CTT TCT-3' y R 5'GAT GTG GCC ATC ACA TTC GTC AGA T-3' en un volumen final de $25 \mu \mathrm{l}$, que contenían 1X PCR buffer (Perkin Elmer), $3 \mathrm{mM} \mathrm{MgCl}$ 0,2, 0,5 mM dNTPs y 1U de Taq polimerasa (Perkin Elmer), con desnaturalización por 1 min a $94^{\circ} \mathrm{C}$ y 30 ciclos con los siguientes pasos $45 \mathrm{~s}$ a $94^{\circ} \mathrm{C}, 1$ minuto a $62^{\circ} \mathrm{C}$ y $1 \min$ a $72^{\circ} \mathrm{C}$, en un termociclador PTC-100 MJ Research. La amplificación produce bandas de $490 \mathrm{pb}$ y $190 \mathrm{pb}$, correspondientes a los alelos I y D respectivamente. Para eliminar la posibilidad que un genotipo ID fuera ampliado como DD, todas las muestras DD fueron reamplificadas con un par de cebadores específicos para la inserción, que reconocen la secuencia insertada, 5'-TGG GAC CAC AGC GCC CGC CAC TAC-3' y 5'-TCG CCA GCC CTC CCA TGC CCA TAA-3', produciendo una banda de aproximadamente 335 pb. ${ }^{12,13}$ Los pasos para esta amplificación consistieron en una desnaturalización inicial de $94^{\circ} \mathrm{C}$ por $1 \mathrm{~min}, 35$ ciclos a $94^{\circ} \mathrm{C}$ por $30 \mathrm{~s}, 56^{\circ} \mathrm{C}$ por $45 \mathrm{~s}, 72^{\circ} \mathrm{C}$ por 2 min y una extensión final a $72^{\circ} \mathrm{C}$ por $7 \mathrm{~min}$.

Para el polimorfismo A1166C del gen AT1R se realizó una PCR anidada, usando para el primer ciclo de amplificación los siguientes cebadores: longF 5'- TCC TCA AAG TCG AGC CCT ACC TCC TAC G -3' y longR 5'- TGA TTT TTG ACC GGG GAA GCT AAA CAT GA 3'-, bajo estas condiciones: $92^{\circ} \mathrm{C}$ por $2 \mathrm{~min}, 35$ ciclos de $94^{\circ} \mathrm{C}$ por $20 \mathrm{~s}, 65^{\circ} \mathrm{C}$ por $30 \mathrm{~s}, 68^{\circ} \mathrm{C}$ por $3 \mathrm{~min}$, y una extensión final de $68^{\circ} \mathrm{C}$ por $20 \mathrm{~min}$. Para la segunda amplificación, se utilizaron los cebadores AT1RF 5'- GCC AAA TCC CAC TCA AAC CTT TCA ACA A- 3', AT1RR 5' AAG CAG GCT AGG GAG ATT GCA TTT CTG T- 3', AT1RA 5'- TCT GCA GCA CTT CAC TAC CAA A TG AAC -3', AT1RC 5'- TCT CCT TCA ATT CTG AAA GTA GCT GAG. Las condiciones fueron: desnaturalización a $94^{\circ} \mathrm{C}$ por $2 \mathrm{~min}$, seguido de 25 ciclos de $94^{\circ} \mathrm{C}$ por $1 \mathrm{~min}, 58^{\circ} \mathrm{C}$ por $1 \mathrm{~min}, 72^{\circ} \mathrm{C}$ por 1 min, y una extensión de $72^{\circ} \mathrm{C}$ por 2 min. 
Los genotipos se visualizaron en geles de agarosa al 1,5\% mediante tinción con Bromuro de Etidio.

Dado que el polimorfismo $+1675 \mathrm{GA}$ del gen AT2R no altera ningún sitio de restricción conocido, la genotipificación se realizó mediante la técnica SSCP (polimorfismo de conformación de cadena sencilla de ADN). La amplificación del gen AT2R se realizó usando los cebadores 1F (5'- ATT ACG TCC CAG CGT CTG AG- 3') y 1R (5'- ATA AAT CAG CTT GCT TAG TGC C- 3'), bajo las siguientes condiciones: $94^{\circ} \mathrm{C}$ por $2 \mathrm{~min}, 30$ ciclos de $94^{\circ} \mathrm{C}$ por $20 \mathrm{~s}, 59^{\circ} \mathrm{C}$ por 35 $\mathrm{s}, 72^{\circ} \mathrm{C}$ por $1 \mathrm{~min}$, y para finalizar una extensión final de $72{ }^{\circ} \mathrm{C}$ por $10 \mathrm{~min}$. Tras la amplificación, se visualizó una banda producto de PCR de $255 \mathrm{pb}$ en geles SSCP a $4^{\circ} \mathrm{C}$ mediante tinción con plata y la interpretación del polimorfismo se hizo según lo reportado previamente, ${ }^{9}$ donde los polimorfismos se clasificaron en GG, GA y AA.

Las variables epidemiológicas medidas fueron edad, peso, talla, antecedente de tabaquismo, gestaciones y paridad, y antecedentes familiares de cáncer. Las variables genéticas fueron los polimorfismos (I/D del gen de la ECA, A1166C del gen AT1R, +1675GA del gen AT2R).

\section{Análisis estadístico}

Los valores son presentados como la media \pm la desviación estándar (DS). El test t de Student se usó para comparar las características antropométricas y sociodemográficas de los dos grupos. El equilibrio Hardy-Weinberg de la frecuencia alélica fue determinado usando el test de Chi-cuadrado. Las diferencias de las frecuencias de los genotipos entre las mujeres con y sin diagnóstico de cáncer fueron determinadas con la prueba de Chi-cuadrado. El nivel de significancia fue de 0,05 y la magnitud de la asociación entre los diferentes genotipos de la ECA y de los receptores de angiotensina II con la presencia del cáncer de mama se expresó en términos de Odds Ratio (OR) y su respectivo intervalo de confianza (IC) del 95\%. Para los polimorfismos, las características categóricas y clínicas fueron tomadas en cuenta. El análisis estadístico se realizó con el programa SPSS versión 17.

\section{RESULTADOS}

Se incluyeron 100 mujeres, 50 casos y 50 controles. Los grupos fueron homogéneos en sus características basales (tabla 1). Ambos grupos presentan antecedentes familiares de cáncer y estos fueron de diferentes orígenes tales como cérvix, mama, colon, piel, pulmón, esófago, hígado entre otros. La distribución genotípica para cada polimorfismo genético en la población se muestra en la tabla 2 . El genotipo ID fue más frecuente en los casos, mientras que el genotipo II, a su vez, fue más frecuente en los controles. En cuanto al polimorfismo en el gen AT1R, los genotipos AA y CC se presentaron con mayor frecuencia en los casos que en los controles, mientras que en los controles predominó el genotipo AC. Para el gen AT2R, únicamente el genotipo GA fue predominante puesto que GG y AA fueron más frecuentes en los controles. Sin embargo,

\begin{tabular}{|l|c|c|c|}
\hline \multicolumn{4}{|c|}{ Tabla 1. Características epidemiológicas de la población de estudio. } \\
\hline \multicolumn{1}{|c|}{ Variables } & $\begin{array}{c}\text { Controles } \\
(\mathbf{n = 5 0 )}\end{array}$ & $\begin{array}{c}\text { Casos } \\
(\mathbf{n = 5 0})\end{array}$ & $\mathbf{p}$ \\
\hline Edad (años) & $52,5 \pm 11,6$ & $50,66 \pm 8,8$ & 0,18 \\
\hline Peso (kg) & $65,4 \pm 14,0$ & $60,74 \pm 12,1$ & 0,082 \\
\hline Talla (m) & $1,57 \pm 0,06$ & $1,55 \pm 0,06$ & 0,09 \\
\hline Tabaquismo & $10(20 \%)$ & $8(16 \%)$ & 0,801 \\
\hline $\mathrm{N}^{\circ}$ gestaciones: promedio del grupo & 1,7 & 2,2 & 0,406 \\
\hline Antecedentes familiares de cáncer & $32(64 \%)$ & $30(60 \%)$ & 0,684 \\
\hline
\end{tabular}




\section{Tabla 2. Distribución genotípica de los polimorfismos evaluados en la población estudiada.}

\begin{tabular}{|l|c|c|c|c|c|}
\hline \multicolumn{1}{|c|}{ Genotipos } & \multicolumn{2}{|c|}{$\begin{array}{c}\text { Controles } \\
(\mathbf{n = 5 0})\end{array}$} & \multicolumn{2}{c|}{$\begin{array}{c}\text { Casos } \\
(\mathbf{n = 5 0})\end{array}$} \\
\hline ECA (I/D) & $\mathbf{n}$ & & $\mathbf{n}$ & & Valor de p \\
\hline ID & 24 & $(48 \%)$ & 23 & $(46 \%)$ & \\
\hline DD & 10 & $(20 \%)$ & 10 & $(20 \%)$ & 0,898 \\
\hline II & 16 & $(32 \%)$ & 17 & $(34 \%)$ & \\
\hline AT1R (A1 166C) & & & & & \\
\hline AC & 21 & $(42 \%)$ & 23 & $(46 \%)$ & \\
\hline AA & 27 & $(54 \%)$ & 26 & $(52 \%)$ & 1 \\
\hline CC & 2 & $(4 \%)$ & 1 & $(2 \%)$ & \\
\hline AT2R (+1675G/A) & & & & & \\
\hline GA & 25 & $(50 \%)$ & 23 & $(46 \%)$ & \\
\hline GG & 10 & $(20 \%)$ & 11 & $(22 \%)$ & 1 \\
\hline AA & 15 & $(30 \%)$ & 16 & $(32 \%)$ & \\
\hline
\end{tabular}

no se encontraron diferencias estadísticamente significativas en la distribución genotípica de los polimorfismos.

En la tabla 3, se muestran las distribuciones alélicas de los polimorfismos estudiados, donde se nota que los alelos D, C y G son los menos frecuentes tanto en los casos como en los controles. Las diferencias no fueron estadísticamente significativas con respecto a los demás alelos en cada polimorfismo $(\mathrm{p}>0,05)$.

De acuerdo a la prueba Chi-cuadrado, el polimorfismo I/D del gen de la ECA y el polimorfismo A1166C del gen AT1R no se encuentra en equilibrio Hardy-Weinberg (I/D $\left.X^{2}=0,230 ; A 1166 C X^{2}=3,00 ; g l=2\right)$. Por el contrario, el polimorfismo +1675GA del gen AT2R en la población presenta una distribución en equilibrio Hardy-Weinberg $\left(X^{2}=21,85\right)$.

En la tabla 4, se muestran las diferentes medidas de asociación entre los genotipos de la ECA y los receptores de la angiotensina II con el cáncer de mama. Ninguno de los genotipos evaluados mostró asociación con el cáncer de mama.

\section{DISCUSIÓN}

Este estudio se realizó debido a la importancia que ha adquirido el SRA en relación con la génesis del cáncer. Se han descrito varios estudios que buscan esclarecer dicha relación, pero los resultados son contradictorios. A este respecto,

Tabla 3. Distribución alélica para los polimorfismos evaluados en la población estudiada.

\begin{tabular}{|c|c|c|c|c|c|c|}
\hline & \multicolumn{2}{|c|}{ ECA (I/D) } & \multicolumn{2}{c|}{ AT1R (A1 166C) } & \multicolumn{2}{c|}{ AT2R (+1675G/A) } \\
\hline & I & D & A & C & G & A \\
\hline Casos & 0,28 & 0,22 & 0,37 & 0,13 & 0,22 & 0,28 \\
\hline Controles & 0,28 & 0,22 & 0,37 & 0,13 & 0,22 & 0,28 \\
\hline Total & 0,56 & 0,44 & 0,74 & 0,26 & 0,44 & 0,56 \\
\hline
\end{tabular}


Tabla 4. Genotipos de ECA, de los receptores AT1R y AT2R en relación al de cáncer de mama.

\begin{tabular}{|l|c|c|c|}
\hline & Casos & Controles & OR (IC 95\%) \\
\hline Genotipos ECA & & & 1 \\
\hline DD & 10 & 10 & $1,08(0,46-2,56)$ \\
\hline ID & 24 & 17 & $0,91(0,37-2,28)$ \\
\hline II & 16 & & \\
\hline Genotipos AT1R & 27 & 26 & $1,08(0,46-2,56)$ \\
\hline AA & 21 & 1 & $0,85(0,36-2,02)$ \\
\hline AC & 23 & 16 & $2,04(0,14-58,95)$ \\
\hline CC & 15 & 23 & $0,96(0,38-2,44)$ \\
\hline Genotipos AT2R & 25 & 11 & $1,17(0,50-2,78)$ \\
\hline AA & 10 & $0,89(0,3-2,57)$ \\
\hline GA & & & \\
\hline GG & & & \\
\hline
\end{tabular}

en un estudio de casos y controles realizado por Haiman y colaboradores, ${ }^{14}$ en el que se determinó la relación entre el riesgo de cáncer de mama y los polimorfismos I/D y A-240T en el gen de la ECA en mujeres japonesas, latinas, afroamericanas y blancas, no encontraron diferencias significativas en la distribución genotípica entre las etnias y plantean que no hay relación entre los polimorfismos descritos y el cáncer de mama. Sin embargo, estos autores observaron una modesta asociación positiva del genotipo II en las mujeres con diagnóstico de cáncer de mama. Por otra parte, Van der Knaap y colaboradores, ${ }^{6}$ en una población de 7.679 mujeres de Roterdam, encontraron que el genotipo predominante en las mujeres con diagnóstico de cáncer fue DD y concluyen que en individuos con ese genotipo los inhibidores del SRA pueden funcionar como protectores contra la entidad. ${ }^{6}$ Por su parte, Koh y colaboradores ${ }^{15}$ en un estudio en una población de mujeres de Singapur, en 258 casos y 670 controles, observaron que las mujeres con el alelo I estaban en un riesgo relativamente menor de presentar cáncer de mama que aquellas con el alelo D. Estos resultados son similares a los encontrados para otras poblaciones. ${ }^{16}$

El genotipo predominante en los casos de nuestra población de estudio fue ID, lo cual se asemeja a lo encontrado en una población de 4.117 mujeres en Róterdam ${ }^{17}$ en el que se evaluó el polimorfismo I/D del gen de la ECA y su relación con el riesgo de cáncer de mama. En este estudio, se encontró que el polimorfismo I/D desempeña un papel importante en el riesgo de este tipo de cáncer, siendo el genotipo ID el más frecuente. Los resultados de nuestro trabajo contrastan también con lo encontrado por Alves y colaboradores en un estudio de casos y controles en una población de mujeres brasileras, ${ }^{8}$ en las cuales el genotipo ID fue el menos frecuente en mujeres con el diagnóstico, lo que le atribuye este fenómeno a una posible codominancia alélica. 
Estas diferencias en los resultados de los estudios pueden ser debidas por una parte al tamaño de la muestra y por otra a diferencias étnicas y de edad (pre y posmenopáusicas), además de otras variables que pueden producir sesgos, como por ejemplo el apareamiento preciso en casos y controles de la exposición ambiental (estrato socioeconómico, dieta, estrés, etc.).

Nuestra población no presenta ninguna relación entre los genotipos del polimorfismo A1166C y el riesgo de cáncer de mama. En los casos, el genotipo más frecuente fue AA, y en los controles el genotipo CC fue el más frecuente. Al igual que en nuestro estudio, Alves y colaboradores ${ }^{8}$ no encontraron relación entre este polimorfismo y el riesgo de cáncer de mama. Asimismo, Gonzalez-Zuloeta ${ }^{16}$ al evaluar en una población de 3.787 mujeres de Róterdam otro polimorfismo para el gen AT1R que se ha descrito relacionado con el cáncer de mama, no reportaron algún papel esclarecido del gen en el riesgo de cáncer de mama, pero sí reportaron el polimorfismo M235T del gen del angiotensinógeno como posible blanco de riesgo de cáncer de mama en mujeres premenopáusicas.

Koh y colaboradores ${ }^{18}$ mencionan que, a partir de su estudio en una población de Singapur en la que evaluaron la relación entre tres polimorfismos en el gen AT1R con el riesgo de cáncer de mama, tanto el gen de la ECA como el gen AT1R son blancos terapéuticos en el tratamiento y prevención del cáncer de mama. En lo concerniente al polimorfismo +1675GA del gen AT2R, en nuestra revisión bibliográfica no encontramos que haya sido evaluado con relación al cáncer de mama, siendo éste el primer estudio en el que se valora dicho polimorfismo con respecto al diagnóstico. Este polimorfismo intrónico ha sido detectado recientemente y está localizado $29 \mathrm{pb}$ antes del inicio del exón 2 del gen AT2R humano, cercano a una región importante para la actividad transcripcional del gen. ${ }^{9}$ Hasta el momento, no se reconoce si esta variación en el gen afecta directamente la transcripción de AT2R; sin embargo, dado que la localización del polimorfismo es en una región involucrada en el control transcripcional de la expresión del gen AT2R, puede esperarse que éste afecte la expresión génica. En un estudio realizado por Imai y colaboradores, ${ }^{19}$ en el que se evaluó el papel del receptor AT1R en el crecimiento tumoral, se concluyó que el bloqueo de la vía de señalización por el receptor AT1R puede llegar a ser un blanco en el tratamiento del cáncer.

\section{CONCLUSIONES}

No se encontró relación entre los polimorfismos estudiados y el cáncer de mama. Teniendo en cuenta las controversias sobre el tema, se considera pertinente realizar estudios de mayor amplitud y rigor metodológico, orientados a establecer el posible papel de los polimorfismos de la ECA en el diagnóstico y manejo del cáncer de mama.

\section{AGRADECIMIENTOS}

A las mujeres que colaboraron voluntariamente para la ejecución de este proyecto. A Oncólogos de Occidente S.A. y al Centro Radiológico del Quindío por facilitarnos el acceso a las pacientes.

\section{REFERENCIAS}

1. Perera NM, Gui GP. Multi-ethnic differences in breast cancer: current concepts and future directions. Int J Cancer 2003;106:463-7.

2. Prichard RS, Hill AD, Dijkstra B, McDermott EW, O'Higgins NJ. The prevention of breast cancer. Br J Surg 2003;90:772-83.

3. Instituto Nacional de cancerología. Cáncer en Cifras. Mortalidad por cáncer según primeras causas y sexo, Colombia 2000-2006. Visitado 2011 Ene 15. Disponible en: http://www.cancer.gov.co/ contenido/contenido.aspx? catID $=437 \& \operatorname{conID}=$ 790\&pagID $=774$

4. Kenrick J. Gene Polymorphisms in the ReninAngiotensin-Aldosterone System and Breast Carcinogenesis: Is there a Connection? TSMJ 2008;9:48-51.

5. Nathanson KL, Wooster R, Weber BL. Breast cancer genetics: What we know and what we need. Nat Med 2001;7:552-6. 
6. Van der Knaap R, Siemes C, Coebergh JW, Van Dujin C, Hofman A, Stricker B. Renin-angiotensin system inhibitors, angiotensin I-converting enzyme gene insertion/deletion polymorphism, and cancer: the Rotterdam Study. Cancer 2008;112:748-57.

7. Fujita M, Hayashi I, Yamashina S, Itoman M, Majima M. Blockade of angiotensin AT1a receptor signaling reduces tumor growth, angiogenesis, and metastasis. Biochem Biophys Res Commun 2002;294:441-7.

8. Alves Corrêa SA, Ribeiro de Noronha SM, Nogueirade-Souza NC, Valleta de Carvalho C, Massad Costa AM, Juvenal Linhares J, et al. Association between the angiotensin-converting enzyme (insertion/deletion) and angiotensin II type 1 receptors (A1166C) polymorphisms and breast cancer among Brazilian women. J Renin Angiotensin Aldosterone Syst 2009; 10:51-8

9. Schmieder RE, Erdmann J, Delles C, Jacobi J, Fleck E, Hilgers K, et al. Effect of the angiotensin II type 2-receptor gene (+1675 G/A) on left ventricular structure in humans. J Am Coll Cardiol 2001;37:175-82.

10. Humphries SE. Cooper, JA, Talmud PJ, Miller GJ. Candidate gene genotypes, along with conventional risk factor assessment, improve estimation of coronary heart disease risk in healthy UK Men. Clin Chem 2007;53:8-16

11. Mendizábal-Ruiz AP, Morales JA, Castro Martinez X, Gutierrez Rubio SA, Valdez L, Vásquez-Camacho JG, et al. RAS polymorphisms in cancerous and benign breast tissue. J Renin Angiotensin Aldosterone Syst 2010. En prensa.

12. Lindpainter K, Pfeffer M, Kreutz R, Stampfer M, Grodstein F, LaMotte F, et al. A prospective evaluation of an angiotensin-converting-enzyme gene polymorphism and the risk of ischemic heart disease. N Engl J Med 1995;332:706-11.

13. Rigoli L, Chimenz R, di Bella C, Cavallaro E, Caruso $\mathrm{R}$, Briuglia S, et al. Angiotensin-converting enzyme and angiotensin type 2 receptor gene genotype distributions in Italian children with congenital uropathies. Pediatr Res 2004;56:988-93.

14. Haiman CA, Henderson SO, Bretsky P, Kolonel LN, Henderson BE. Genetic variation in angiotensin I-converting enzyme (ACE) and breast cancer risk: the multiethnic cohort. Cancer Res 2003;63:6984-87.

15. Koh WP, Yuan JM, Sun CL, van den Berg D, Seow A, Lee HP, et al. Angiotensin I-converting enzyme (ACE) gene polymorphism and breast cancer risk among Chinese women in Singapore. Cancer Res 2003;63:573-8.

16. González-Zuloeta AM, Arias-Vásquez A, Siemes C, Yazdanpanah M, Coebergh JW, Hofman A, et al. Differential roles of Angiotensinogen and Angiotensin Receptor type 1 polymorphisms in breast cancer risk. Breast Cancer Res Treat 2007;101:299-304.

17. González-Zuloeta AM, Arias Vásquez A, SayedTabatabaei FA, Coebergh JW, Hofman A, Njajou O, et al. Angiotensin-converting enzyme gene insertion/ deletion polymorphism and breast cancer risk. Cancer Epidemiol Biomarkers 2005;14:2143-6.

18. Koh WP, Yuan JM, Van Den Berg D, Lee HP, Yu MC. Polymorphisms in angiotensin II type 1 receptor and angiotensin I-converting enzyme genes and breast cancer risk among Chinese women in Singapore. Carcinogenesis 2005;26:459-64.

19. Imai N, Hashimoto T, Kihara M, Yoshida S, Kawana I, Yazawa T, et al. Roles for host and tumor angiotensin II type 1 receptor in tumor growth and tumor-associated angiogenesis. Lab Invest 2007;87:189-98.

Conflicto de intereses: ninguno declarado.

Fuente de financiación: proyecto financiado por la Universidad del Quindío. Joven investigador cofinanciado por COLCIENCIAS, contrato 110 de 2008. 